\title{
Global Estimates of Errors in Quantum Computation by the Feynman-Vernon Formalism
}

\author{
Erik Aurell ${ }^{1,2,3}$
}

Received: 30 August 2017 / Accepted: 13 April 2018 / Published online: 18 April 2018

(C) The Author(s) 2018

\begin{abstract}
The operation of a quantum computer is considered as a general quantum operation on a mixed state on many qubits followed by a measurement. The general quantum operation is further represented as a Feynman-Vernon double path integral over the histories of the qubits and of an environment, and afterward tracing out the environment. The qubit histories are taken to be paths on the two-sphere $S^{2}$ as in Klauder's coherent-state path integral of spin, and the environment is assumed to consist of harmonic oscillators initially in thermal equilibrium, and linearly coupled to to qubit operators $\hat{S}_{z}$. The environment can then be integrated out to give a Feynman-Vernon influence action coupling the forward and backward histories of the qubits. This representation allows to derive in a simple way estimates that the total error of operation of a quantum computer without error correction scales linearly with the number of qubits and the time of operation. It also allows to discuss Kitaev's toric code interacting with an environment in the same manner.
\end{abstract}

Keywords Noisy quantum computing · Feynman-Vernon method

\section{Introduction}

Quantum computers are physical devices that manipulate quantum states to execute information-processing tasks $[46,49]$. To build a general-purpose quantum computer is a difficult experimental challenge where several different realizations have been proposed since the 1990ies $[28,41]$. While several large commercial initiatives to reach on the order of 50

Erik Aurell

eaurell@kth.se

1 Department of Computational Science and Technology, KTH - Royal Institute of Technology, AlbaNova University Center, 10691 Stockholm, Sweden

2 Department of the Applied Physics and Computer Science, Aalto University, P.O. Box 11100, 00076 Aalto, Finland

3 Institute of Theoretical Physics, Chinese Academy of Sciences, P.O. Box 2735, Beijing 100190, China 
qubits have been widely reported on recently $[18,56]$, the current public state-of-the-art is that around ten qubits can be manipulated in the lab in a manner approaching to what would be required for a general-purpose quantum computer [37]. For more restricted computational tasks a device using about 1000 qubits has been reported to lead to important speed-up over classical algorithms [19].

Quantum computation would, if successful, upend the characterization of what is possible and not possible in classical information processing. That is its main promise, but also a main difficulty since a large quantum computer must have a substantial number of degrees of freedom, and large physical systems have a strong tendency to turn classical [31]. The underlying mechanism of this quantum-to-classical transition is the decoherence of the quantum state by interactions between the quantum computer and the rest of the world [67].

There has been a long-running polemic against the possibility of quantum computing going back at least to [6]. This paper is primarily concerned with the critique recently put forward in [34], to be discussed again in Sects. 2 and 3 below. The gist of these arguments is that the standard error models considered in the quantum computing literature are too restrictive, and that quantum computing will for this reason not be possible in the real world when errors may be correlated in time and space. Indeed, errors in quantum computation are often discussed as mis-application of operators, which indicates a kind of classical uncertainty in the external potential controlling the quantum system, and not errors induced by coupling to another (unobserved) quantum system.

The goal of this paper is to consider this problem from a global point of view by investigating the errors caused by coupling a system of spins to a thermal bath of bosonic degrees of freedom. Such errors can be correlated over arbitrary distances at low enough temperature, but in a specific way determined by the physical interaction. The analysis is carried out by combining a coherent-state path integral representation of the dynamics of spin systems with the Feynman-Vernon method to integrate out the bath. The effect of the bath is then described by an influence functional coupling forward and backward coherent-state path integrals. This influence functional can be estimated simply when the system-bath interaction is weak.

The main result is that the errors usually considered are not an idealization but rather a worst-case scenario. It is correct that these standard error models mostly disregard correlations in time and space, but a physical heat bath, which could generate such correlations, is a comparatively simple system, and not an adversary in the sense of complexity theory. For the paradigmatic example of the Kitaev toric code it is further showed that coupling to a bath has effects exponentially small in the size of the lattice. For such a system only the errors normally considered therefore need to be corrected.

The paper is organized as follows: in Sect. 2 I position the problem in relation to the quantum computational literature and in Sect. 3 I state "pessimistic hypothesis" of Kalai in a version suitable to be analyzed by the methods used herein. In Sect. 4 I review quantum noise, and in Sect. 5 I introduce the Feynman-Vernon double path integral as a model of quantum operations on $n$ qubits which also interact linearly with a bath of harmonic oscillators. The system qubit histories are formulated using Klauder's coherent-state path integral for spin [39, 58], and the Feynman-Vernon action (after integrating out the bath) are therefore interference terms between the forward and backward coherent-state paths. In Sects. 6 and 7 I discuss two simple models where each qubit is either connected to its own environment, or where all the qubits are connected to one and the same environment. In Sect. 8 I treat Kitaev's toric code interacting with the same kind of environment, and in Sect. 9 I summarize the paper. Appendices contain standard material on Klauder's path integral and the Feynman-Vernon theory, an annotated discussion of the pessimistic hypothesis as formulated in [34], and a discussion of quantum error correction and recovery, included for completeness. 


\section{Preliminaries and Relation to the Literature}

The operation of an ideal quantum computer without decoherence can be cast in a language similar to information theory where the elementary operations (quantum gates) are implemented as unitary transformations on a pure quantum state, acting on a few qubits at a time $[9,21]$. The errors made in a quantum computation due to decoherence were first discussed quantitatively by Aharonov, Kitaev and Nisan in [3] using a formalism where the state is a density matrix and the transformations are quantum operations mapping density matrices to density matrices. This standard formalism is outlined in Sect. 3 below. In [3] the quantum operations were patterned after unitary quantum gates and assumed to factorize both over operations and between qubits which are not acted upon by the same operation. The computational power of such a quantum computer can hence be expressed as properties of networks of "noisy quantum gates". The main conclusion of [3] is that if a quantum computer of this type makes $L$ quantum operations (operates $L$ quantum gates in total, counted with multiplicity) each with error $\epsilon$, then the combined error of the total operation scales as $L \epsilon$. This estimate leads to higher accuracy needed the larger the quantum computer. It therefore points to the need for quantum error correction to make quantum computation possible $[25,38,48]$.

Quantum or classical error correction must be formulated relative to some error model. It is for instance trivially impossible to correct the errors caused by a transmission channel which completely forgets the initial state. An important class of error models in quantum information theory, which will be discussed again below in Sects. 3 and 4, assumes that the quantum operation has a block structure where each block acts on the states of one qubit [20,59]. Such an error model is hence local both in space (physical qubits) and in time (no memory). Furthermore, in the same context it is often assumed that errors are Pauli channels which describe mis-application of operators. The analysis of such error models have been generalized to probability distribution over channel histories $[12,60]$, which are therefore no longer local in time, but still local in space.

The originally proposed purpose of quantum computing was to simulate another quantum system of interest. Feynman argued that in general this cannot be done with a classical device [23]; experimental and theoretical progress in this direction of research was recently reviewed in [27]. Quantum supremacy is a term for similar efforts formulated in the language of computational complexity theory $[32,44,53]$. The objective is then to solve some classically impossible computational problem using quantum resources, or to show mathematically that an output of some quantum device needs many more resources to be simulated classically. A central model problem has here been BosonSampling [1], related to computing the permanent of a matrix. It was shown already in [61] that the permanent appears in an exact expression for the probability of scattering bosons, and that it therefore can be estimated by an ideal quantum device. In contrast, while the determinant of matrix can be computed in polynomial time e.g. by diagonalization, all general classical algorithms to compute a permanent take exponential time in the size of the matrix.

Noisy BosonSampling is the task of sampling from the distribution of a number of nonideal scattered bosons (photons), whether or not that distribution is related to a permanent, or has some other application [50]. Aaronson and Arkhipov showed that Noisy BosonSampling remains computationally hard for somewhat abstract and small noise [1], while Kalai and Kindler showed that it becomes simple when the scattering matrix is perturbed by another small but fixed matrix [36]. Closer to the physics of the problem Aaronson and Brod showed that Noisy BosonSampling is hard when at most a finite number of the photons are dropped [2] while Oszmaniec and Brod showed that it is easy if out of $n$ photons all but 
$\sqrt{n}$ are dropped [47]. It is currently unknown whether Noisy BosonSampling is hard or easy when a constant fraction $(\alpha n)$ of $n$ photons are dropped.

Noisy BosonSampling mainly lies outside of the issues studied in this paper because photon drop, as a quantum problem, is on the level of second quantization. The focus in the following will be on systems composed of a fixed number of spin- $\frac{1}{2}$ fermions, or "qubits", as has been the case in most of the quantum information theory literature. As briefly reviewed in Sect. 4 such systems can also behave noisily in various ways, but for the most part the computational entities can be assumed to be long-lived, and the analysis can therefore be carried out on the level of first quantization.

\section{Statement of the Problem}

We assume that there are physical systems with Hilbert space of dimension two that we call qubits. A pure quantum state on $n$ qubits is a complex ray in a $N=2^{n}$-dimensional Hilbert space. A mixed state on the same $n$ qubits is a density matrix $\rho$ which is a non-negative Hermitian operator of unit trace; the set of all density matrices has dimension $N^{2}-1$. A quantum operation is a linear map from a set of density matrices to a set of density matrices which we will write $\Phi$; this is a set of dimension $N^{4}-N^{2}$. The geometry of density matrices and general operations of a single qubit $(n=1, N=2)$ are well understood as the Bloch sphere and linear transformations of the Bloch sphere, but for higher dimensions there is no such simple picture [10].

Let now $n$ qubits start in the pure state $|i>=| i_{1}, \ldots, i_{n}>$, density matrix $\rho_{i}=$ $\left|i_{1}, \ldots, i_{n}\right\rangle\left\langle i_{1}, \ldots, i_{n}\right|$, and let there be a unitary quantum operation $\Phi_{0} \rho_{i}=U \rho_{i} U^{\dagger}$ with the property that if the qubits are measured in the final state then the Boolean vector $\mathbf{f}=\left(f_{1}, \ldots, f_{k}\right)$ is observed with probability $P_{i f}^{(0)}=\left\langle f\left|\Phi_{0} \rho_{i}\right| f\right\rangle$. Let then the system be coupled to an environment and described by a quantum operation $\Phi$ and corresponding probabilities $P_{i f}=\left\langle f\left|\Phi \rho_{i}\right| f\right\rangle$. A basic measure of the error of $P_{i f}$ with respect to $P_{i f}^{(0)}$ is the variational distance between the two probability distributions:

$$
\mathrm{TVD}=\sum_{\mathbf{f}}\left|P_{i f}-P_{i f}^{(0)}\right|
$$

Any choice of final observable $O$ taking values $o$ leads to probability distributions $P_{i o}$ and $P_{i o}^{(0)}$, and it can be shown the maximum of (1) over $O$ is the trace norm $\|\cdot\|_{1}$ of the difference of the corresponding density matrices. Furthermore, the authors of [3] introduced the diamond norm over super-operators and prove the important inequalities $\left\|\Phi_{1} \Phi_{2}\right\|_{\diamond} \leq\left\|\Phi_{1}\right\|_{\diamond}\left\|\Phi_{2}\right\|_{\diamond}$ (Lemma 12, statement 3) and $\|\Phi \rho\|_{1} \leq\|\Phi\|_{\diamond}\|\rho\|_{1}$ (Lemma 12, combining statements 1 and 2). For two series of quantum operations that can be written

$$
\Phi=\Phi_{L} \Phi_{L-1} \cdots \Phi_{1} \quad \Phi^{(0)}=\Phi_{L}^{(0)} \Phi_{L-1}^{(0)} \cdots \Phi_{1}^{(0)}
$$

and where each pair of unitary and noisy quantum operations satisfies $\left\|\Phi_{l}-\Phi_{l}^{(0)}\right\|_{\diamond}<\epsilon$ this leads to [3, Theorem 4]

$$
\mathrm{TVD} \leq L \cdot \epsilon
$$

Equation (3) says that the total error of a quantum computer scales linearly with the number of operations $L$ which in many realistic settings would be proportional to the number of computational units $(n)$ and the duration of the process $(t)$. If an error rate is defined as TVD per $n$ and $t$ then (3) has the interpretation that the error rate per quantum operation of a 
quantum computer is bounded by a constant. In particular it does not increase with the number of qubits the quantum computer is operating on. Quantum error correction systems are built on physical (small-scale) qubits which are used to build logical (larger-scale) qubits on which the quantum computation is done. Under assumptions that will be critically discussed in Sect. 4, the linear scaling (3) together with sufficiently small error-rate for individual physical qubits and gates acting on them allows quantum computing based on quantum error-correction.

Kalai's "pessimistic hypothesis" [34] is the contrary position to the above. For concreteness I will formulate it as follows:

Pessimistic hypothesis for a large enough quantum computational system it is not possible to maintain the scaling of the error in (3) due to correlations of errors in space and time.

If true, this would render high-quality quantum error-correction in particular, and quantum computing in general impossible for large enough systems. At this point it must be emphasized that the above statement cannot be found in [34], or in its extended version available on-line as [35], or in Kalai's earlier papers such as [33]. It is a reformulation of the pessimistic hypothesis such that the methods used in this paper are applicable. I argue in Appendices A and B that it is a reasonable reformulation. In Appendix B I also discuss briefly a model proposed [33] which can be contrasted to exact equations found by Weiss, Grifoni, Hänggi and co-workers [30].

The problem addressed in this paper is to discuss bounds on the left-hand side of (3) without considering error rates of individual components at all. The objective is hence to circumvent the critique of [34] by treating the problem as one of the physics of open quantum system, and not as one of quantum information theory. The tool to do this, used in Sects. 5-8, is the Feynman-Vernon formalism. As measurements would usually be performed in some predetermined way which would often more or less amount to measuring the $z$-components of all the qubits I will for simplicity assume a given initial state $\rho_{i}$ and a given final observable, and consider all the variability of the problem to stem from $\Phi$ being different from $\Phi^{(0)}$. The trace norm and the diamond norm will therefore not appear in the following analysis. Furthermore, error rate is not a concept intrinsic to quantum mechanics. In the following error rate will therefore only be discussed as an auxiliary quantity defined in terms of TVD, in the same way as done above, in text below equation (3).

\section{Quantum Noise}

As quantum noise is central to the problem addressed in this paper I will in this Section make a detour and outline the theoretical and experimental boundaries within which I discuss this concept. The reader primarily interested in the main argument may proceed directly to Sect. 5 .

Quantum mechanics is based on unitary evolution of a state between measurements and non-unitary collapse of the wave function when it is measured. The latter is a source of uncertainty which is taken to be a basic property of the world [66]. Quantum computing without decoherence fully incorporates this quantum mechanical measurement uncertainty which therefore does not need to be considered further here.

Unitary time evolution shares with Hamiltonian dynamics in classical mechanics the property that it is deterministic and time-reversal invariant. It is therefore, in a colloquial sense of the word, noise-free, and does not, by itself, explain the subjective human experience that time flows forward towards the future, and not towards the past. Modifications of the 
equations of quantum mechanics to be stochastic were considered in [29] and more recently discussed by Weinberg $[62,64]$. The success of quantum mechanics as physical theory implies that such modifications, if they exist, must be very small. Such hypothetical modifications can therefore also be ignored in the present context; Weinberg in [63] gives a relative bound of $10^{-17}$ by comparing to the stability of atomic clocks.

The issue of quantum noise is instead that a quantum mechanical system may effectively develop in a different manner than by unitary time evolution because it is interacting with another (unobserved) system. There are two ways in which this can be described: by quantum operations acting on the density matrix of the system, as summarized above in Sect. 3, or by explicitly modeling the time evolution of the observed and unobserved systems together. It is well known that every quantum operation has an environmental representation but that this is not unique; many environments and couplings to the environment correspond to the same quantum operation on the system [10].

From the point of view of information theory the simplest and most natural quantum noise models are the quantum operations that are structurally simple and most similar to unitary evolution. The factorized error model in [3] outlined in Sect. 3 assumes that the elementary unitary transformation of a noise-free quantum gate is modified to a quantum operation that acts non-trivially only on these same qubits. A more physical interpretation was given in [60] where each qubit is attached to its own separate environment (a "bath"), and then extended to the case where these baths interact when and in the same combinations as the qubits do. Other contributions have extended the model and methods of [3] to when the quantum operations depend on time $[11,12,54,60]$. All these contributions (and others) have in common a high level of mathematical sophistication, and the need for assumptions that are physically questionable, or at least not simple.

From the point of view of physics the simplest and most natural quantum noise models are instead those that result from simple interactions with simple environments. The simplest of these are linear interactions with an environment of harmonic oscillators. This is the model that will be introduced in Sect. 5 below and used as the basis of the subsequent analysis. Such models describe a system interacting with delocalized degrees of freedom such as photons (in cavity electrodynamics) or phonons (in solid state systems).

It is worth emphasizing that the two views on simplicity are not aligned; in fact they are more nearly orthogonal. The quantum operation that results from a harmonic oscillator bath originally in a thermal state depends strongly on bath temperature. If sufficiently high then the resulting time development of the system is Markovian i.e. factorizes over time, one of the assumptions made in [3]. In this same limit the system however behaves nearly classically $[16,17]$, not a desired property of a quantum computing device. If on the other hand bath temperature is low then the noise from the bath acting on the system will be moderate in overall size but correlated in time and space. In the regime where a system could work as a quantum computer it must thus be able to deal with such non-trivial noise, at least as long as it may be interacting with phonons or photons.

The current leading technology for future quantum computers are coupled superconducting quantum circuits $[22,65]$. Each logical element (qubit) is then in fact formed by a mesoscopic object containing many millions of atoms, but where the behavior of one degree of freedom can be assimilated to that of one quantum spin. A figure-of-merit of how accurate is such a description is the ratio between the gate time of operation and the qubit relaxation time for which the current experimental (published) record for coherent super-positions is about $5 \cdot 10^{-4}$. This is based on $T_{2} \sim 20 \mu \mathrm{s}$ and a previously established cycle time about $10 \mathrm{~ns}$, alternatively one can give the number $2 \cdot 10^{-4}$ based on the qubit relaxation time $T_{1} \sim 60 \mu \mathrm{s}$ [51]. The current (published) record for a system of nine qubits, and with all 
properties measured in the same system, is for one qubit (one out of nine) about $10^{-3}$. This number is based on measured relaxation times $T_{1}=18-41 \mu s$ and measured operation times $20-45 n s$, as given in [37, Table S3].

The qubit degree of freedom in the quantum circuit interacts with the other degrees of freedom in the circuit, with degrees of freedom in the surrounding device and material, and with as external control potential, an influence also mediated by the degrees of freedom of the device. The total dynamics is hence potentially quite complex. Deviations from desired dynamics include changes in density matrix of computational states of the qubit as well as leakage, i.e. excitations of higher non-computational states of the qubit. Considering only the first type of effects they can be modeled by interactions between a qubit and an environment, the kind of model to be introduced in Sect. 5 below. As recently reviewed in [52], fast environmental modes have to be treated quantum mechanically while slow environmental modes can be treated as classical random fields. The analysis in Sect. 5 and following hence pertain to the fast environmental modes, treated as a harmonic oscillator bath interacting linearly with the qubit.

The influence of classical random fields on the density matrix of a qubit will be a a superposition of random unitary transformations i.e. $\rho \rightarrow V_{i} \rho V_{i}^{\dagger}$, each unitary $V_{i}$ applied with probabilities $p_{i}$. On a single qubit all such transformations can be represented as Pauli channels i.e. $\rho \rightarrow p_{0} \rho+\sum_{i} p_{i} \hat{\sigma}^{i} \rho \hat{\sigma}^{i}$ where $\hat{\sigma}^{i}$ are the Pauli matrices, and $\left(p_{0}, p_{1}, p_{2}, p_{3}\right)$ are non-negative numbers that sum to one (take $V_{i}=e^{i \frac{\pi}{2} \hat{\sigma}_{i}}=i \hat{\sigma}_{i}$ ). A qubit system perturbed by a Pauli channel is one of the standard models in the quantum computing literature [20,25,38,54], and the factorized error model in [3] is obviously also of the same general kind. As follows from the preceding discussion such models are not realistic descriptions of interactions with an environment: as they have no memory the corresponding environmental modes should be treated quantum mechanically. On the other hand, Pauli channels and similar models describe the effects on the quantum system of a memory-less classical uncertainty in the control potential. Note in passing that the number of independent unitary transformations in $N$-dimensional Hilbert space is $N^{2}-1$, and the dimensionality of the class of random super-positions is thus only a $1 / N^{2}$-small fraction of all quantum operations. For instance, all random superposition of unitary transformations are unital (preserve the identity) and therefore do not include e.g. amplitude decay channels [10].

The kind of error models considered in the more recent quantum information literature which include memory $[11,12,60]$ are more aligned with the influence of slow environmental modes. However, $1 / f$-noise is an ubiquitous property of solid state devices, and this may lead stronger memory effects than have been analyzed up to now, for further discussion the interested reader is referred to [52].

Summarizing this Section, quantum noise in systems currently considered for quantum computing can be classified as (A) classical noise acting quantum mechanically, (B) influence from a slow quantum environment that can be described classically, and (C) influence from a fast quantum environment that has to be described quantum mechanically. From a fundamental point of view only (C) can be an obstacle to quantum, as opposed to classical, computing. From a practical and experimental point of view any of (A), (B) and (C) could be the main problem. Most of the quantum information literature tacitly assume (A), and as will be shown in the following analysis it is correct that $(\mathrm{C})$ generally gives weaker effects than (A). The effects of (B) are more difficult to treat, likely more system dependent, and could well be main obstacles to successful quantum computing, as argued in [52]. 


\section{The Open Quantum System Model}

The aim of this section is to compare the two probabilities $P_{i f}$ and $P_{i f}^{(0)}$ in one term in (1) when the quantum computer interacts with a heat bath. The Hamiltonian describing the quantum computer and the bath together is

$$
\hat{H}=\hat{H}_{S}+\hat{H}_{I}+\hat{H}_{B}
$$

where $\hat{H}_{S}$ depends only on the variables describing the quantum computer, from hereon also referred to as the system, $\hat{H}_{B}$ depends only on the bath variables, and $\hat{H}_{I}$ describes the interaction of the system and the bath. We first consider the system without the heat bath and use the observation that any unitary transformation can be implemented by unitary transformation acting on at most two qubits at a time [9,21]. The system Hamiltonian will thus be

$$
\hat{H}_{S}=\hbar \sum_{a} \mu_{a} \mathbf{S}^{a}+\hbar \sum_{a b} \mathbf{S}^{a} \kappa_{a b} \mathbf{S}^{b}
$$

where $\mathbf{S}^{a}=\left\{\hat{S}_{x}^{a}, \hat{S}_{a}^{y}, \hat{S}_{a}^{z}\right\}$ are the spin operators acting on the $a$ 'th qubit, $\mu_{a}$ is a 3 -vector and $\kappa_{a b}$ is a 3-by-3 matrix. Both the $\mu$ 's and the $\kappa$ 's have dimension frequency and can depend on time as required to implement the overall unitary transformation

$$
U=\mathcal{T} e^{-\frac{i}{\hbar} \int \hat{H}_{S} d t}
$$

where $\mathcal{T}$ means time ordering. Following the prescription of [39] we insert an over-complete resolution of the identity and write

$$
\begin{aligned}
\langle\mathbf{f}|U| \mathbf{i}\rangle= & \int \prod_{a} \frac{\sin \theta_{a}^{(i)} d \theta_{a}^{(i)} d \phi_{a}^{(i)}}{2 \pi} \frac{\sin \theta_{a}^{(f)} d \theta_{a}^{(f)} d \phi_{a}^{(f)}}{2 \pi} \\
& \left\langle\mathbf{f} \mid \theta^{(f)}, \phi^{(f)}\right\rangle K_{c s}\left(\theta^{(f)}, \phi^{(f)}, \theta^{(i)}, \phi^{(i)}\right)\left\langle\theta^{(i)}, \phi^{(i)} \mid \mathbf{i}\right\rangle
\end{aligned}
$$

where $\left(\theta_{a}^{(i)}, \phi_{a}^{(i)}\right)$ and $\left(\theta_{a}^{(f)}, \phi_{a}^{(f)}\right)$ parametrize unit spheres, $K_{c s}$ is the coherent-state propagator and $\left|\theta^{(i)}, \phi^{(i)}\right\rangle$ and $\left|\theta^{(f)}, \phi^{(f)}\right\rangle$ are the initial and final product coherent states. The unitary quantum operation is given by

$$
\Phi_{0} \rho=U \rho U^{\dagger}
$$

and the first matrix element we are looking for is

$$
\begin{aligned}
P_{i f}^{(0)}= & \int \prod_{a} \frac{\sin \theta_{a}^{(f, F)} d \theta_{a}^{(f, F)} d \phi_{a}^{(f, F)}}{2 \pi} \frac{\sin \theta_{a}^{(f, B)} d \theta_{a}^{(f, B)} d \phi_{a}^{(f, B)}}{2 \pi}\left\langle\mathbf{f} \mid \theta^{(f, F)}, \phi^{(f, F)}\right\rangle\left\langle\theta^{(f, B)}, \phi^{(f, B)} \mid \mathbf{f}\right\rangle \\
& \int \prod_{a} \frac{\sin \theta_{a}^{(i, F)} d \theta_{a}^{(i, F)} d \phi_{a}^{(i, F)}}{2 \pi} \frac{\sin \theta_{a}^{(i, B)} d \theta_{a}^{(i, B)} d \phi_{a}^{(i, B)}}{2 \pi}\left\langle\mathbf{i} \mid \theta^{(i, B)}, \phi^{(i, B)}\right\rangle\left\langle\theta^{(i, F)}, \phi^{(i, F)} \mid \mathbf{i}\right\rangle \\
& K_{c s}\left(\theta^{(f, F)}, \phi^{(f, F)}, \theta^{(i, F)}, \phi^{(i, F)}\right) K_{c s}^{*}\left(\theta^{(f, B)}, \phi^{(f, B)}, \theta^{(i, B)}, \phi^{(i, B)}\right)
\end{aligned}
$$

where $F$ means "forward" and $B$ means "backward". The coherent-state propagator has a path integral representation

$$
K_{c S}=\lim _{\epsilon \rightarrow 0} \mathcal{N}_{\epsilon} \int \prod_{a} \mathcal{D} \theta_{a} \mathcal{D} \phi_{a} e^{i \int \mathcal{L}_{k i n}+\epsilon \mathcal{L}_{r e g}+\mathcal{L}_{S} d t}
$$

where $\mathcal{L}_{\text {kin }}$ and $\mathcal{L}_{\text {reg }}$ are Klauder's kinetic and regularization terms, $\mathcal{N}_{\epsilon}$ is a normalization, and $\mathcal{L}_{S}$ represents the interactions. The two coherent state propagators in (9) can therefore 
be written as a double path integral over forward and backward paths. I summarize for convenience the Klauder theory in Appendix $\mathrm{C}$ where I also give the explicit expression for $\mathcal{L}_{S}$ corresponding to (5). For compactness I shall write (9) as

$$
P_{i f}^{(0)}=\langle\mathbf{1}\rangle_{i f}
$$

where $\langle\cdots\rangle_{\text {if }}$ is a shorthand the averages implied by (9) and (10).

To the above model we now add a bath described by $\hat{H}_{B}$ and $\hat{H}_{I}$. The bath and the system are originally assumed to be in a product state $\rho_{i}^{T O T}=\rho_{i} \oplus \rho_{i}^{B}$, and the final total state is $\rho_{f}^{T O T}=U^{T O T} \rho_{i}^{T O T}\left(U^{T O T}\right)^{\dagger}$ where $U^{T O T}$, analogous to $U$ in (6), depends on the whole Hamiltonian. The reduced density matrix of the system only at the final time is $\rho_{f}=\operatorname{Tr}_{B}\left[\rho_{f}^{T O T}\right]$. The total unitary operator $U^{T O T}$ can be represented as a path integral over the forward paths of both the system and the bath, and analogously for $\left(U^{T O T}\right)^{\dagger}$. The initial density matrix of the bath $\rho_{i}^{B}$ can also be represented as a function of the starting points of the forward and backward paths of the bath (both to be integrated over).

As was first shown in [24] the bath variables can then be integrated out. Instead of the two coherent-state propagators in (9), each expressed as a separate path integral (10), we then instead have

$$
\begin{aligned}
K_{F V}= & \lim _{\epsilon \rightarrow 0}\left|\mathcal{N}_{\epsilon}\right|^{2} \int \prod_{a} \mathcal{D} \theta^{F} \mathcal{D} \phi^{F} e^{i \int \mathcal{L}_{k i n}\left(\theta^{F}, \phi^{F}\right)+\epsilon \mathcal{L}_{r e g}\left(\theta^{F}, \phi^{F}\right)+\mathcal{L}_{S}\left(\theta^{F}, \phi^{F}\right) d t} \\
& \int \prod_{a} \mathcal{D} \theta^{B} \mathcal{D} \phi^{B} e^{-i \int \mathcal{L}_{k i n}\left(\theta^{B}, \phi^{B}\right)-\epsilon \mathcal{L}_{r e g}\left(\theta^{B}, \phi^{B}\right)-\mathcal{L}_{S}\left(\theta^{B}, \phi^{B}\right) d t} e^{i \Phi_{F V}}
\end{aligned}
$$

where

$$
\Phi_{F V}=\Phi_{F V}\left[\theta^{F}, \phi^{F}, \theta^{B}, \phi^{B}\right]
$$

is the Feynman-Vernon influence action. We can then write the second matrix element we are looking for as

$$
P_{i f}=\left\langle e^{i \Phi_{F V}}\right\rangle_{i f}
$$

When the Feynman-Vernon influence action is relatively small we therefore have

$$
P_{i f}-P_{i f}^{(0)} \approx\left\langle i \Phi_{F V}\right\rangle_{i f}
$$

Extracting a representative value $\overline{\Phi_{F V}}$ we have

$$
\operatorname{TVD}=\sum_{\mathbf{f}}\left|P_{i f}-P_{i f}^{(0)}\right| \approx \overline{\Phi_{F V}}
$$

Eq. (16) is the first result of this paper. It means that the error made by the whole system is determined by a global description of the system and the environment and is proportional to the strength of the interaction between the two. It therefore allows to estimate the scaling of the error with system size by estimating the scaling of $\Phi_{F V}$. In the following two section we will look at two simple models where this leads to the same scaling as Aharonov-Kitaev-Nisan (eq. 3).

\section{The Spin-Boson Model with One Bath Per Spin}

The first model of one spin interacting with the environment was the spin-boson model, extensively investigated in [42]. We are here concerned with general interacting spin systems 
and therefore use a different representation of spin histories than in [42], but the description of the bath and the coupling of the system and the bath will be the same. The model discussed here will hence be referred to as the spin-boson model with one bath per spin ("1 - 1 "). For one spin the terms $\hat{H}_{I}$ and $\hat{H}_{B}$ in (4) are [42]

$$
H_{\text {spin-boson }}^{1-1}=\sum_{n} \hbar \omega_{n}\left(a_{n}^{\dagger} a_{n}+\frac{1}{2}\right)+\hat{S}_{z}\left(\sum_{n} \sqrt{\frac{\hbar}{2 m_{n} \omega_{n}}} C_{n} a_{n}+\text { c.c. }\right)
$$

where $\hat{S}_{z}$ is the $z$-component of the spin and $a_{n}^{\dagger}$ and $a_{n}$ and the creation and annihilation operators of harmonic oscillator labeled by index $n$. The mass and the frequency of the harmonic oscillators are given by $m_{n}$ and $\omega_{n}$, and the strength of the interaction between the spin and the environment is given by $C_{n}$. For many spins the model discussed in this section assumes one set of terms as in (17) per spin, each with a different set of operators and $a_{n}^{\dagger}$ and $a_{n}$

In the path integral formulation we write instead of (17) the classical Hamiltonian representing the terms involving the environment as

$$
H_{\text {bath }}=\sum_{n} \frac{1}{2 m_{n}} p_{n}^{2}+\frac{1}{2} m_{n} \omega_{n}^{2} x_{n}^{2}+S_{z} \sum_{n} C_{n} x_{n}
$$

where in the coherent-state path integral $S_{z}$ is the function $\frac{1}{2} \cos \theta$, as discussed in Appendix C. The Feynman-Vernon functional in (13) can then be computed explicitly as a functional of the forward and backward spin histories, as outlined in Appendix D. We will here only need the estimate of the Feynman-Vernon action for one spin coupled to one bath given in (57) and that the Feynman-Vernon actions from more than one disconnected systems add. The total Feynman-Vernon action in (13) is then estimated as

$$
\Phi_{F V} \sim \eta \cdot n \cdot\left(t_{f}-t_{i}\right)
$$

where $n$ is the number of spins, $\eta$ is an overall measure of the strength of the interaction between a spin and its bath, and $t_{f}-t_{i}$ is the duration of the process. Following (16) and assuming weak coupling (small $\eta$ ) we then have

$$
\mathrm{TVD} \sim \eta \cdot n \cdot\left(t_{f}-t_{i}\right)
$$

Eq. (16) is the second result of this paper. It should be read as a generalization (3) to a definite physical model where the interaction strength $\eta$ is what gives rise to the elementary error $\epsilon$, and where the number of qubits times the duration of the process $\left(n \cdot\left(t_{f}-t_{i}\right)\right)$ plays the role of of the number of "noisy operations" $L$. While there are similarities there are also differences. In the model used in [3] time does not enter since the system is supposed to develop unitarily between the "noisy operations". That is a somewhat unphysical assumption as any quantum system will interact with the environment to some extent, and therefore decohere continuously. On the other hand, in the model considered here the complexity of the quantum operation that implements the computational task does not enter; all else equal it does not matter how many operations are performed in the same time window as long as the form and the strength of the interaction between each qubit and its bath remains the same.

\section{The Spin-Boson Model with One Common Bath for All Spins}

A model where each spin has its own bath supposes that each spin is located in a separate material with separate delocalized degrees of freedom. Although not inconceivable one may 
also consider the situation where all the spins are located in the same material and interacting with the same delocalized degrees of freedom. The interaction terms between the spins and one oscillator in the common bath are then described by the action

$$
S\left[x_{b},\left\{\theta_{k}^{f}\right\}\right]=\frac{1}{2} \sum_{k} \int_{t_{i}}^{t_{f}} C_{b} x_{b}(t) \cos \theta_{k}^{f} d t
$$

where the interaction coefficients $C_{b}$ for simplicity have been taken the same for all spins interacting with the same bath oscillator. We can re-write the right-hand side of (21) as $\frac{n}{2} \int_{t_{i}}^{t_{f}} d t C_{n} x_{n}(t) \overline{\cos \theta^{f}}(t)$ where $n$ is the number of spins (qubits) and $\overline{\cos \theta^{f}}=\frac{1}{n} \sum_{k} \cos \theta_{k}^{f}$, and same for the backward path, and then integrate out the bath oscillators. The result will be a Feynman-Vernon influence functional of the two collective coordinates of the same structure as (52) and (53), and which can be written

$$
\Phi=n^{2} \frac{i}{\hbar} S_{i}\left[\overline{\cos \theta^{f}}, \overline{\cos \theta^{b}}\right]-n^{2} \frac{1}{\hbar} S_{r}\left[\overline{\cos \theta^{f}}, \overline{\cos \theta^{b}}\right]
$$

Formally (22) scales quadratically with number of spins (qubits). However, it is physically reasonable that an increasing number of spins in the same material would take more place. One may think of either the spins are arranged along a line, or arranged on two-dimensional grid. In the first case one dimension of the system increases proportional to $n$ while in the second case two dimensions of the system increase proportional to $\sqrt{n}$, and in both cases the interaction coefficient $C$ between the isolated spin and a delocalized mode can be expected to scale as $1 / n$. The number of modes in a small frequency interval $d \omega$ will increase as $n$ and the overall bath power spectrum $J(\omega)$ therefore decreases as $1 / n$. Combining these estimates one gets back the linear scaling in (20). Furthermore, the differences between the forward and backward paths are fluctuating quantities and at least in the high-temperature near-classical regime investigated in [17] one can expect $\overline{\cos \theta^{f}}-\overline{\cos \theta^{b}}$ to scale as $1 / \sqrt{n}$. The real part of $\Phi$ in (22) would therefore give a contribution independent of the number of qubits while the imaginary part of $\Phi$ in (22) would give an error increasing slower than linearly.

\section{The Toric Code in the Feynman-Vernon Theory}

A canonical model of quantum computing and quantum error correction is Kitaev's toric code [38]. In the simplest version, which will be considered here, an $N \times M$ lattice of spins are located at edges in a regular lattice on the 2-torus, and operated on by operators called stabilizers

$$
A_{s}=\prod_{i \in \operatorname{star}(i)} \sigma_{i}^{x} \quad B_{p}=\prod_{i \in \partial p} \sigma_{i}^{z}
$$

In $A$-type stabilizers $i \in \operatorname{star}(i)$ denote the spins (edges) in the neighborhood of a vertex $s$ and in $B$-type stabilizers $i \in \partial p$ denote the spins (edges) around a plaquette $p ; \sigma_{i}^{z}$ and $\sigma_{i}^{x}$ are Pauli operators acting on spin $i$. All the stabilizers commute and the eigenspace of all of them measured simultaneously is four-dimensional. This Hilbert space can be identified with that of two spins, usually in this context called logical qubits, and Pauli operators on these two qubits are products of operators on the physical spins taken around the two basic circuits on the torus. Note that we are here concerned with the Kitaev code, and not the closely related quantum statistical mechanical system known as the Kitaev model. In that second case, see Eq. 29 below, the operators in Eq. 29 are terms in a Hamiltonian operator, and not measured 
continuously. The four-dimensional ground state of the Kitaev model is the one where the eigenvalues of $A_{s}$ and $B_{p}$ in Eq. 29 are all equal to one.

More complex versions of toric codes which can accommodate many more qubits will not be considered further here, nor the very considerable experimental challenges of actually building such systems; for a recent review, see [25]. The system under consideration hence consists of $N M$ physical spins and a bath of harmonic oscillators developing according to (4) where in addition the stabilizers are continuously measured. The system Hamiltonian is thus

$$
H_{S}=H_{S}\left(s_{1}^{x}, s_{1}^{y}, s_{1}^{z}, s_{2}^{x}, s_{2}^{y}, s_{2}^{z}\right)
$$

where

$$
\begin{aligned}
& s_{1}^{x}=\prod_{i \in C_{1}} \sigma_{i}^{x} \quad s_{1}^{z}=\prod_{i \in C_{2}^{\prime}} \sigma_{i}^{z} \quad s_{1}^{y}=i s_{1}^{z} s_{1}^{x} \\
& s_{2}^{x}=\prod_{i \in C_{2}} \sigma_{i}^{x} \quad s_{2}^{z}=\prod_{i \in C_{1}^{\prime}} \sigma_{i}^{z} s_{2}^{y}=i s_{2}^{z} s_{2}^{x}
\end{aligned}
$$

are the Pauli operators acting on the logical qubits, and $C_{1}, C_{2}$ and $C_{1}^{\prime}, C_{2}^{\prime}$ are the two basic cycles of the torus in respectively the vertex-centered and plaquette-centered lattice.

A basis of the states of the physical spins is $\left|i_{1}, i_{2}, \ldots, i_{N M}\right\rangle$ where $i_{p}= \pm 1$ denotes the up (down) state of spin $p$. An alternative basis is by above given by the $k$ values of the $z$-components of logical qubits $l_{r}$ (here $k=2$ ) and the $N M-k$ values of the stabilizers $m_{q}= \pm 1$. These two bases are related by a unitary transformation

$$
\left|\mathbf{l}, \mathbf{m}>=\sum_{\mathbf{i}} A_{\mathbf{i}}^{\mathbf{l}, \mathbf{m}}\right| \mathbf{i}>
$$

where $\mid \mathbf{l}, \mathbf{m}>$ denotes $\mid l_{1}, \ldots, l_{k}, m_{1}, \ldots, m_{N M-k}>$ and $\mid \mathbf{i}>$ denotes $\mid i_{1}, i_{2}, \ldots, i_{N M}>$. By orthogonality of the states of the stabilizers and the logical qubits we have

$$
\sum_{\mathbf{i}} A_{\mathbf{i}}^{\mathbf{l}, \mathbf{m}}\left(A_{\mathbf{i}}^{\mathbf{I}^{\prime}, \mathbf{m}^{\prime}}\right)^{*}=\mathbf{1}_{l, l^{\prime}} \mathbf{1}_{m, m^{\prime}}
$$

Now assume that over some stretch of time the measured values of all the stabilizers are constant. These are then not histories of quantum variables but known classical (and constant) records. The interaction of the physical spins with one bath oscillator $b$ gives an interaction Hamiltonian for the logical qubits

$$
\begin{aligned}
Q\left(\mathbf{l}, \mathbf{l}^{\prime} ; \mathbf{m}\right) & =\left\langle\mathbf{l}^{\prime}, \mathbf{m}\left|\sum_{r} \hat{\sigma}_{r}^{z}\right| \mathbf{l}, \mathbf{m}\right\rangle \\
& =\sum_{\mathbf{i}} A_{\mathbf{i}}^{\mathbf{l}, \mathbf{m}}\left(A_{\mathbf{i}}^{\mathbf{l}^{\prime}, \mathbf{m}}\right)^{*}\left(\sum_{r}(-1)^{i_{r}}\right)
\end{aligned}
$$

From these follow interaction Hamiltonians for the coherent-state representations of the histories of the logical qubits in the forward and backward paths

$$
\begin{aligned}
& Q^{F}\left(\theta^{F}, \phi^{F} ; \mathbf{m}\right)=\sum_{\mathbf{l}, \mathbf{I}^{\prime}}\left\langle\theta^{F}, \phi^{F} \mid \mathbf{I}^{\prime}\right\rangle Q\left(\mathbf{l}, \mathbf{I}^{\prime} ; \mathbf{m}\right)\left\langle\mathbf{l} \mid \theta^{F}, \phi^{F}\right\rangle \\
& Q^{B}\left(\theta^{B}, \phi^{B} ; \mathbf{m}\right)=\sum_{\mathbf{l}, \mathbf{I}^{\prime}}\left\langle\theta^{B}, \phi^{B} \mid \mathbf{I}^{\prime}\right\rangle Q\left(\mathbf{l}, \mathbf{I}^{\prime} ; \mathbf{m}\right)\left\langle\mathbf{l} \mid \theta^{B}, \phi^{B}\right\rangle
\end{aligned}
$$


These more complicated functions $Q^{F}$ and $Q^{B}$ play the same role for the interaction of the toric code with a bath of oscillators as the sums of the cosines in the simple model discussed in Sect. 7 above, compare Eq. (21).

The influence functional is as above estimated as

$$
\Phi_{F V} \sim \eta \bar{Q}^{2}\left(t_{f}-t_{i}\right)
$$

where $\bar{Q}$ is a typical value of $Q^{F}$ and $Q^{B}$. A rough estimate of $\bar{Q}$ follows from assuming that each element of $A$ in $(25)$ is about $2^{-\frac{N M}{2}}$ with a fluctuating sign, which is consistent with (26). $Q\left(\mathbf{l}, \mathbf{I}^{\prime} ; \mathbf{m}\right)$ in (27) is then the sum of $2^{N M}$ terms of fluctuating signs, each of size about $2^{-N M}$ and hence of overall typical size $2^{-\frac{N M}{2}}$. Each of the two functions $Q^{F}$ and $Q^{B}$ is then a sum of $\left(2^{k}\right)^{2}$ such terms multiplied by the matrix elements with the angles which each have RMS average $2^{-\frac{k}{2}}$ (see Appendix C). The approximate sizes of $Q^{F}$ and $Q^{B}$ are hence $2^{\frac{k-N M}{2}}$, the amplitude $\bar{Q}^{2}$ in (28) is consequently $2^{k-N M}$, and the influence of the bath on the states of the logical qubits exponentially small in system size.

A more systematic estimate of $\bar{Q}$ follows from observing that $Q\left(\mathbf{l}, \mathbf{I}^{\prime} ; \mathbf{m}\right)$ is the matrix element of the operators coupling the system to the heat bath between two eigenstates the Kitaev model given by Hamiltonian

$$
H_{K}=-\sum_{s} A_{s}-\sum_{p} B_{p}
$$

The two states have the same quantum numbers (m) determined by the eigenvalues of the operators $A_{s}$ and $B_{p}$, and the same or different quantum numbers given by the logical operators acting on the logical qubits $\left(\mathbf{l}\right.$ and $\left.\mathbf{l}^{\prime}\right)$. It is known that the matrix elements of local operators in the ground state of the Kitaev model are exponentially small in system size [14]. For $(\mathbf{m})=(1,1,1, \ldots, 1)$ the logical qubits of the toric code are therefore almost insensitive to interactions with bath.

The results of this section are positive for the Kitaev code, and it may be useful to compare other results in the literature. First, the standard view is that the Kitaev model in 2D with Hamiltonian (29) does not preserve its state when interacting with a finite-temperature heat bath $[4,5,13,15,20]$, a result often stated as that the Kitaev model is not a stable quantum memory. That is not the same setting as considered here, as the stabilizer operators are then not continuously measured. To reproduce these results in the formalism of the present paper one should promote the measured values of the stabilizers $\mathbf{m}$ to be quantum variables represented in a larger coherent-state path integral by forwards and backwards angles $\left\{\theta^{\mathbf{m}, F} \phi^{\mathbf{m}, F}\right\}$ and $\left\{\theta^{\mathbf{m}, B} \phi^{\mathbf{m}, B}\right\}$. Instead of (27) we then have (m and $\mathbf{m}$ ' different)

$$
\begin{aligned}
Q\left(\mathbf{l}, \mathbf{m}, \mathbf{l}^{\prime}, \mathbf{m}^{\prime}\right) & =\left\langle\mathbf{l}^{\prime}, \mathbf{m}^{\prime}\left|\sum_{r} \hat{\sigma}_{r}^{z}\right| \mathbf{l}, \mathbf{m}\right\rangle \\
& =\sum_{\mathbf{i}} A_{\mathbf{i}}^{\mathbf{l}, \mathbf{m}}\left(A_{\mathbf{i}}^{\mathbf{l}^{\prime}, \mathbf{m}^{\prime}}\right)^{*}\left(\sum_{r}(-1)^{i_{r}}\right)
\end{aligned}
$$

which we can again estimate as $2^{-\frac{N M}{2}}$. The two terms $Q^{F}$ and $Q^{B}$ are however now sums of $\left(2^{N M}\right)^{2}$ terms, and are therefore not small in system size. In this case estimate (28) hence gives essentially the same result as (20). 


\section{Discussion}

In this work I have considered the error made by a quantum computer weakly coupled to an environment such that the quantum computer cannot be meaningfully described as a network of "noisy quantum gates". I have instead estimated the error by combining Klauder's path integral for spin and a Feynman-Vernon elimination of a thermal bath modeled as a set of harmonic oscillators interacting linearly with the qubits.

I have looked at three models. In the first two all qubits are computational units and all interact directly with a heat bath as in the spin-boson model [42]. In these two simpler models no error correction was considered: the goal was to see if the scaling of the overall error found by Aharonov, Kitaev and Nisan in [3] needs to be modified. The answer is negative. Instead of the error rate of a noisy quantum gate, a concept not defined for these models, the crucial parameter is the interaction strength between the system and the heat bath. If that parameter is small the total error scales at most linearly with system size (number of qubits) and time of operation - without any assumptions on locality in space and time.

The third model considered in the toric code of Kitaev [38] in 2D where additionally the physical qubits interact with a heat bath as in the spin-boson model. The computational units (logical qubits) of this model are non-localized degrees of freedom, much fewer in number than the physical qubits. The analysis brings out the fact that the states of the logical qubits are almost insensitive to interactions with a bath, at least in the ground state of the related Kitaev model where all the stabilizers (defined above) have value one. One consequence of this observation is that such an influence does not need to be corrected, as it is exponentially small in the system size. The combination of Klauder's path integral and Feynman-Vernon allows to treat together the interaction with a heat bath and other errors that can be modeled as Pauli channels, and can hence be considered an alternative to the quantum semi-group dynamics (Davies generator formalism) within which many systematic studies of this and the related Kitaev model have be performed previously $[4,5,13]$.

A thermal bath consisting of harmonic oscillators is a model of delocalized environmental modes such as phonons. The main degrees of freedom in a real material at very low temperature, such as defects and nuclear spins, are on the other hand likely to be localized, and may be more accurately described as a spin bath [55]. For this case it may be argued that the environment of each qubit consists in a finite set of neighboring spins the effects of which would in principle also be given by a Feynman-Vernon action as in (13). Although precise estimates of this action would be more difficult to obtain, there seems to be no reason to assume that the number of environmental spins interacting with one qubit scales with the number of qubits of the quantum computer.

Finally, although the analysis is this paper has shown that fast environmental modes that have to be treated quantum mechanically are not a fundamental problem for quantum computing, there remains slow environmental modes. As long as these may be treated classically they cannot be a problem for quantum computing per se, but may nevertheless still pose very significant obstacles in practice, a point of view forcefully argued in [52].

Acknowledgements I thank Gil Kalai for a stimulating discussion and Yuri Galperin, Benjamin Huard, Petteri Kaski, Cris Moore, Sorin Paraoanu, Sergey Pershoguba and Karol Życzkowski for constructive remarks. This research has been supported by the Swedish Science Council through Grant 621-2012-2982, by the Academy of Finland through its Center of Excellence COIN, and by the Chinese Academy of Sciences CAS President's International Fellowship Initiative (PIFI) Grant No. 2016 VMA002.

Open Access This article is distributed under the terms of the Creative Commons Attribution 4.0 International License (http://creativecommons.org/licenses/by/4.0/), which permits unrestricted use, distribution, and 
reproduction in any medium, provided you give appropriate credit to the original author(s) and the source, provide a link to the Creative Commons license, and indicate if changes were made.

\section{Appendix A: A Quantum-Mechanical Formulation of Kalai's $\epsilon$}

The purpose of this appendix is to argue that the total variational distance as defined above in (1), counted per qubit, is a reasonable quantum mechanical interpretation of the error rate $\epsilon$ discussed in [34]. I emphasize that this interpretation can not be found [34], but is introduced here as a way to state the problem within the theory of open quantum systems.

To do so we consider the special case where the quantum operation $\Phi_{0}$, determined by a unitary transformation $U$, is such that there is a single final state $|f\rangle$ with $\left\langle f\left|\Phi_{0} \rho_{i}\right| f\right\rangle=1$. Applying the quantum operation $\Phi_{0}$ to $|i\rangle$ can then be said to yield $|f\rangle$ with certainty, and $\Phi_{0}$ can then be called "noise-less". Applying the "noisy" quantum operation $\Phi$ and measuring all the qubits would on the other hand give the Boolean vector $\mathbf{f}$ with probability $1-\epsilon^{\prime}$ for some $\epsilon^{\prime}>0$ and a result different from $\mathbf{f}$ with total probability $\epsilon^{\prime}$. Let now further $\Phi$ be such that the probabilities $p(\tilde{f})$ are sensibly different from zero only when the Hamming distance between $\tilde{f}$ and $\mathbf{f}$ is at most one, i.e. when at most one qubit has been flipped, and let the probability to flip any one qubit be $\epsilon=\epsilon^{\prime} / n$. The error rate so defined is then the same as $\frac{1}{2}$ TVD, where TVD is defined in (1).

\section{Appendix B: Kalai’s Pessimistic Hypothesis}

The purpose of this appendix is to argue that Kalai's pessimistic hypothesis claims that $\epsilon$ as introduced above in Appedix A scales linearly with number of qubits in the quantum computer. The argument proceeds by selected quotes from [34]. We start from

The error rate in every realization of a universal quantum circuit scales up (at least) linearly with the number of qubits

Readers of [34] will note that this statement is followed by

The effort required to obtain a bounded error level for any implementation of universal quantum circuits increases (at least) exponentially with the number of qubits

which is also important to Kalai's argument concerning universal quantum computers. In the present discussion, which focuses on the consequences for open quantum systems, I will however limit myself to the first part.

In [34] Kalai also argues by the example of a depolarizing one-qubit channel described by

$$
\Phi \rho=(1-p) \rho+p \frac{1}{2} \mathbf{1}
$$

where $\rho$ is the density matrix of a qubit (a positive Hermitian 2-by-2 matrix of unit trace), $\Phi$ is the quantum operation (a linear operator of the set of such matrices on itself) and $\frac{1}{2} \mathbf{1}$ is the completely depolarized density matrix. The error rate is then taken to be $p$ and, more generally

...error rate can be defined as the probability that a qubit is corrupted at a computation

step, conditioned on it surviving up to this step

which is followed by 
...when we say that the rate of noise per qubit scales up linearly with the number of qubits, we mean that when we double the number of qubits in the circuit, the probability for a single qubit to be corrupted in a small time interval doubles

In combination the above quotes imply that Kalai's pessimistic hypothesis states that the total error of the whole system scales at least quadratically with the number of qubits. In the interpretation used here, see Appendix A above, this is taken to mean that the total variational distance in (1) also scales at least quadratically. Similarly to the main text and Appendix A I emphasize again that this quadratic global scaling cannot be found [34] but is a consequence of the further interpretations introduced here.

The word pessimistic reflects the differences in focus between a physical and a computerscience style of analysis, alluded to from another point of view in Sect. 4 in main text. In physics one is (at least most of the time) not interested in worst-case scenarios or adversarial reasoning, but in typical behaviour of plausible models. This difference can be illustrated by considering the special case that the qubits interact among themselves only through polynomials in $\sigma_{z}$ operators, and are acted upon by terms linear in $\sigma_{x}$ (but not $\sigma_{y}$ ). As a computational device this would represent quantum annealing [8], hence somewhat restrictive, but the development of the system including interaction with a bath as in this paper can then be treated fully as the spin-boson problem [42]. The path-integral representation would involve only piece-wise constants paths. For this setting it is known that the expectation values of spin operators, equivalent for one spin to the full knowledge of the density matrix, obey exact (though complicated) generalized master equations (GME) presented e.g. in [30, Sect. 11.2.2]. The time-smoothened noise operators proposed by Kalai in e.g. Eq. 1 in [33] appear different in structure from GME. Arguments built on such hypothetical operators are therefore not persuasive in a context of definite physical models of the system-bath interaction, as is the focus of this paper.

\section{Appendix C: The Klauder Coherent-State Path Integral for Spin}

This appendix summarizes properties pertaining to the Klauder coherent-state path integral. The coherent states are defined as

$$
|\theta, \phi\rangle=e^{-i \phi \hat{S}_{z}} e^{-i \theta \hat{S}_{y}}|\uparrow\rangle=\left(\begin{array}{c}
e^{-\frac{i}{2} \phi} \cos \frac{\theta}{2} \\
e^{\frac{i}{2} \phi} \sin \frac{\theta}{2}
\end{array}\right)
$$

The two angles $\theta \in[0, \pi]$ and $\phi \in[0,2 \pi]$ parametrize the unit sphere with area $4 \pi$. The various matrix elements used in the main text and below are hence

$$
\langle\uparrow \mid \theta, \phi\rangle=e^{-\frac{i}{2} \phi} \cos \frac{\theta}{2} \quad\langle\downarrow \mid \theta, \phi\rangle=e^{\frac{i}{2} \phi} \sin \frac{\theta}{2}
$$

and the matrix element between two coherent states is

$$
\begin{aligned}
\left\langle\theta^{\prime}, \phi^{\prime} \mid \theta, \phi\right\rangle & =\left\langle\theta^{\prime}, \phi^{\prime} \mid \uparrow\right\rangle\langle\uparrow \mid \theta, \phi\rangle+\left\langle\theta^{\prime}, \phi^{\prime} \mid \downarrow\right\rangle\langle\downarrow \mid \theta, \phi\rangle \\
& =\cos \frac{\phi^{\prime}-\phi}{2} \cos \frac{\theta^{\prime}-\theta}{2}+i \sin \frac{\phi^{\prime}-\phi}{2} \cos \frac{\theta^{\prime}+\theta}{2}
\end{aligned}
$$

When the two sets of angles are close this matrix element is

$$
<\theta^{\prime}, \phi^{\prime} \mid \theta, \phi>\approx 1+\frac{i}{2}\left(\phi^{\prime}-\phi\right) \cos \theta
$$


up to terms which are small as $\left(\phi^{\prime}-\phi\right)^{2}$ and $\left(\theta^{\prime}-\theta\right)^{2}$. Matrix elements of the operator for the $z$-component of spin are

$$
\begin{aligned}
\left\langle\theta^{\prime}, \phi^{\prime}\left|\hat{S}_{z}\right| \theta, \phi\right\rangle=\left\langle\theta^{\prime}, \phi^{\prime} \mid \uparrow\right\rangle\left\langle\uparrow\left|\hat{S}_{z}\right| \uparrow\right\rangle\langle\uparrow \mid \theta, \phi\rangle & \\
& +\left\langle\theta^{\prime}, \phi^{\prime} \mid \uparrow\right\rangle\left\langle\uparrow\left|\hat{S}_{z}\right| \downarrow\right\rangle\langle\downarrow \mid \theta, \phi\rangle \\
& +\left\langle\theta^{\prime}, \phi^{\prime} \mid \downarrow\right\rangle\left\langle\downarrow\left|\hat{S}_{z}\right| \uparrow\right\rangle\langle\uparrow \mid \theta, \phi\rangle \\
& \quad+\left\langle\theta^{\prime}, \phi^{\prime} \mid \downarrow\right\rangle\left\langle\downarrow\left|\hat{S}_{z}\right| \downarrow\right\rangle\langle\downarrow \mid \theta, \phi\rangle \\
= & \frac{1}{2}\left(\cos \frac{\phi^{\prime}-\phi}{2} \cos \frac{\theta^{\prime}+\theta}{2}+i \sin \frac{\phi^{\prime}-\phi}{2} \cos \frac{\theta^{\prime}-\theta}{2}\right)
\end{aligned}
$$

which when the two sets of angles are close means

$$
\left\langle\theta^{\prime}, \phi^{\prime}\left|\hat{S}_{z}\right| \theta, \phi\right\rangle \approx \frac{1}{2} \cos \theta
$$

Similarly

$$
\begin{aligned}
\left\langle\theta^{\prime}, \phi^{\prime}\left|\hat{S}_{x}\right| \theta, \phi\right\rangle & \approx \frac{1}{2} \sin \theta \cos \phi \\
\left\langle\theta^{\prime}, \phi^{\prime}\left|\hat{S}_{x}\right| \theta, \phi\right\rangle & \approx \frac{1}{2} \sin \theta \sin \phi
\end{aligned}
$$

The vector $\mathbf{S}=\frac{1}{2}(\sin \theta \cos \phi, \sin \theta \sin \phi, \cos \theta)$ is the radial vector of length $\frac{1}{2}$, polar angle $\theta$ and azimuthal angle $\phi$. The coherent states provide a partition of the unity in an over-complete basis. Using (33) we have

$$
\begin{aligned}
\langle\uparrow \mid \uparrow\rangle & =\int \frac{\sin \theta d \phi d \theta}{2 \pi}\langle\uparrow \mid \theta, \phi\rangle\langle\theta, \phi \mid \uparrow\rangle=1 \\
\langle\uparrow \mid \downarrow\rangle & =\int \frac{\sin \theta d \phi d \theta}{2 \pi}\langle\uparrow \mid \theta, \phi\rangle\langle\theta, \phi \mid \downarrow\rangle=0 \\
\langle\downarrow \mid \uparrow\rangle & =\int \frac{\sin \theta d \phi d \theta}{2 \pi}\langle\downarrow \mid \theta, \phi\rangle\langle\theta, \phi \mid \uparrow\rangle=0 \\
\langle\downarrow \mid \downarrow\rangle & =\int \frac{\sin \theta d \phi d \theta}{2 \pi}\langle\downarrow \mid \theta, \phi\rangle\langle\theta, \phi \mid \downarrow\rangle=1
\end{aligned}
$$

A time evolution operator can therefore be expressed as

$$
\begin{aligned}
\mathcal{T} e^{-\frac{i}{\hbar} \int \hat{H} d t}= & \prod_{n} \int \frac{\sin \theta_{n} d \phi_{n} d \theta_{n}}{2 \pi} \cdots\left|\theta_{n+1}, \phi_{n+1}\right\rangle \\
& \left\langle\theta_{n+1}, \phi_{n+1}\left|e^{-\frac{i}{\hbar} \int_{t_{n}}^{t_{n+1}} \hat{H} d t}\right| \theta_{n}, \phi_{n}\right\rangle \\
& \left\langle\theta_{n}, \phi_{n}\right| \cdots
\end{aligned}
$$


If it can be arranged that two consecutive sets of angles are close, the interaction term (10) for the interaction Hamiltonian (5) is, using (36) and (37),

$$
\begin{aligned}
\mathcal{L}_{S}= & -\frac{1}{2} \sum_{a} \mu_{a}^{z} \cos \theta_{a}+\left(\mu_{a}^{x} \cos \phi_{a}+\mu_{a}^{y} \sin \phi_{a}\right) \\
& -\frac{1}{4} \sum_{a b} \kappa_{a b}^{z z} \cos \theta_{a} \cos \theta_{b}+\cos \theta_{a} \sin \theta_{b}\left(\kappa_{a b}^{z x} \cos \phi_{b}\right. \\
& \left.+\kappa_{a b}^{z y} \sin \phi_{b}\right)+\left(\kappa_{a b}^{x z} \cos \phi_{a}+\kappa_{a b}^{y z} \sin \phi_{a}\right) \sin \theta_{a} \cos \theta_{b} \\
& +\sin \theta_{a} \sin \theta_{b}\left(\kappa_{a b}^{x x} \cos \phi_{a} \cos \phi_{b} \kappa_{a b}^{x y} \cos \phi_{a} \sin \phi_{b}\right. \\
& \left.+\kappa_{a b}^{y x} \sin \phi_{a} \cos \phi_{b}+\kappa_{a b}^{y x} \sin \phi_{a} \cos \phi_{b}++\mu_{a}^{y} \sin \phi_{a}\right) \\
& \left(\mu_{b}^{x} \cos \phi_{b}+\mu_{b}^{y} \sin \phi_{b}\right)
\end{aligned}
$$

where the factor $\hbar$ has been included for convenience, compare (5) and (10). For the discussion below and in the main text it only matters that (40) is some definite function of the angles parametrizing the spin history.

To enforce that two consecutive sets of angles are close one uses a regularization term

$$
\left.\mathcal{L}_{\text {reg }}=\frac{1}{2}\left(\dot{\theta}^{2}+\sin ^{2} \theta \dot{\phi}^{2}\right)\right)
$$

The matrix element (34) can then be written

$$
\left\langle\theta^{\prime}, \phi^{\prime} \mid \theta, \phi\right\rangle \approx e^{i \int_{t}^{t^{\prime}} \mathcal{L}_{k i n}}
$$

defining the kinetic term in (10):

$$
\mathcal{L}_{k i n}=\frac{1}{2} \cos \theta \dot{\phi}
$$

The regularization and interaction terms in (10) are as in (41) and (40) above. The canonical momenta conjugate to $\theta$ and $\phi$ are

$$
p_{\theta}=\epsilon \dot{\theta} \quad p=p_{\phi}=\epsilon \sin ^{2} \theta \dot{\phi}+\frac{1}{2} \cos \theta
$$

As discussed in [39] and in [7], when $\epsilon$ is set to zero the remaining action is first order. The function $\frac{1}{2} \cos \theta$ then takes the meaning of momentum $p$, conjugate to $\phi$. and the radial vector $\mathbf{S}$ can be written $\left(\frac{1}{2} \sqrt{1-4 p^{2}} \cos \phi, \frac{1}{2} \sqrt{1-4 p^{2}} \sin \phi, p\right)$. The Poisson brackets of the components of this vector satisfy the angular momentum relations. This suggests that in the path integral the operators $\hat{S}_{x}, \hat{S}_{y}$ and $\hat{S}_{z}$ should be translated into these functions $S_{x}, S_{y}$ and $S_{z}$, which is indeed the prescription given by (36), (37), (38) and (40).

With the regularization (41) the path integral is a standard one, and $\epsilon$ could be interpreted as the mass of a particle confined to move on the surface of a sphere of fixed radius. The path integral in curved space (as is the sphere) is a well-developed topic with several complexities [57], but for the present discussion, where the "mass term" is only for regularization, one can simply interpret the integral of (41) as a time discretization

$$
\epsilon \int_{t_{i}}^{t_{f}} \mathcal{L}_{r e g} \approx \sum_{n} \frac{\epsilon}{2 \Delta t_{n}}\left(\Delta \theta_{n}^{2}+\overline{\sin ^{2} \theta} \Delta \phi_{n}^{2}\right)
$$

and where $\overline{\sin ^{2} \theta}$ indicates $e . g$. mid-point prescription and the normalizing coefficient is

$$
N_{\epsilon}=\prod_{n}\left(\overline{\sin ^{2} \theta_{n}}\right)^{\frac{1}{2}} \frac{\epsilon}{2 \pi i \Delta t_{n}}
$$


The regularization and the normalization are a weight on the Fourier components of the spin history, and the path integral built on a discretization of (43) and (41) can therefore be written

$$
\int \prod_{k} d \mu_{\epsilon}\left(\hat{\theta}_{k}, \hat{\phi}_{k}\right) e^{i \int \mathcal{L}_{k i n}\left[\left\{\hat{\theta}_{k}, \hat{\phi}_{k}\right\}\right]}
$$

For finite $\epsilon$ this weight penalizes high Fourier components. Consider two realizations $\left\{\hat{\theta}_{k}, \hat{\phi}_{k}\right\}$ and $\left\{\hat{\theta}^{\prime}{ }_{k}, \hat{\phi}^{\prime}{ }_{k}\right\}$ which coincide at the two endpoints. The difference of their actions

$$
\int \mathcal{L}_{k i n}\left[\left\{\hat{\theta}_{k}^{\prime}, \hat{\phi}_{k}^{\prime}\right\}\right]-\int \mathcal{L}_{k i n}\left[\left\{\hat{\theta}_{k}, \hat{\phi}_{k}\right\}\right]
$$

is the integral of $\mathcal{L}_{\text {kin }}$ around a closed path, which in turn equals the area on the surface of the sphere circumscribed by that path [7]. This area depends only weakly on high Fourier components and the limit of zero $\epsilon$ is therefore well-behaved.

\section{Appendix D: The Feynman-Vernon Method for Spin Histories}

The starting point is the interaction and bath Hamiltonian for a single spin interacting with a bath:

$$
H_{\text {bath }}+H_{\mathrm{I}}=\sum_{n} \frac{1}{2 m_{n}} p_{n}^{2}+\frac{1}{2} m_{n} \omega_{n}^{2}\left(x_{n}+S_{z} \frac{C_{n}}{m_{n} \omega_{n}^{2}}\right)^{2}
$$

In above $S_{z}$ is read $\frac{1}{2} \cos \theta$, the function representing the operator $\hat{S}_{z}$ in the Klauder path integral. The last term from expanding the squares in (49), $\sum_{n} S_{z}^{2} \frac{1}{2} \frac{C_{n}}{m_{n} \omega_{n}^{2}}$, only depends on the spin history, and is a counter-term which it has become customary to include in the interaction term [17]. The two variables $x_{n}$ and $p_{n}$ are the coordinate and momentum of bath oscillator $n$ with mass $m_{n}$ and frequency $\omega_{n}$. It is assumed that each bath oscillator is initially at thermodynamic equilibrium independent of the spin i.e. relative to

$$
H_{\text {bath }}=\sum_{n} \frac{1}{2 m_{n}} p_{n}^{2}+\frac{1}{2} m_{n} \omega_{n}^{2} x_{n}^{2}
$$

The bath oscillators can then be integrated out and the Feynman-Vernon influence functional is

$$
i \Phi=\frac{i}{\hbar} S_{i}[\cdot]-\frac{1}{\hbar} S_{r}[\cdot]
$$

where

$$
\begin{aligned}
& S_{i}=\int^{t_{f}} \int^{t}\left(S_{z}^{f}(t)-S_{z}^{b}(t)\right)\left(S_{z}^{f}(s)+S_{z}^{b}(s)\right) k_{i} \\
& S_{r}=\int^{t_{f}} \int^{t}\left(S_{z}^{f}(t)-S_{z}^{b}(t)\right)\left(S_{z}^{f}(s)-S_{z}^{b}(s)\right) k_{r}
\end{aligned}
$$

The kernels $k_{i}$ and $k_{r}$ depend on the bath spectral density

$$
J(\omega)=\pi \sum_{n} \frac{C_{n}^{2}}{m_{n} \omega_{n}} \delta\left(\omega-\omega_{n}\right)
$$


as

$$
\begin{aligned}
k_{i} & =\frac{1}{\pi} \int_{0}^{\infty} J(\omega) \sin \omega(t-s) \\
k_{R} & =\frac{1}{\pi} \int_{0}^{\infty} J(\omega) \cos \omega(t-s) \operatorname{coth}\left(\frac{\hbar \omega}{2 k_{B} T}\right)
\end{aligned}
$$

where $T$ is the temperature of the bath. In (52) and (53) for the the forward spin history $S_{z}^{f}=\frac{1}{2} \cos \theta^{f}$ and for the backward $S_{z}^{b}=\frac{1}{2} \cos \theta^{b}$. It is a consequence of the form of the coupling in the spin-boson model that the Feynman-Vernon action only depends on the polar angle $\theta$ and not on the azimuthal angle $\phi$.

Assuming that $J(\omega)$ behaves as $\eta \omega^{s} \omega_{c}^{-s-1}$ up to some large frequency $\Omega$ and decays quickly for larger frequencies [42] $k_{i}$ and $k_{r}$ will both be proportional to $\eta$ (in units of $\omega_{c}$ ). The kernel $k_{i}$ will have support on a time interval of width $\Omega^{-1}$ and the kernel $k_{r}$ will have support on a time interval of width the larger of $\Omega^{-1}$ and $\hbar / k_{B} T$. Both (52) and (53) can therefore be simply estimated as

$$
S_{i}, S_{r} \sim \eta\left(t_{f}-t_{i}\right)
$$

where $t_{f}-t_{i}$ is the duration of the process. This estimate is used above in the main text.

\section{Appendix E: Analysis of Recovery and Quantum Error Correction}

In the main text of the paper quantum error correction was not considered. The purpose of this appendix is to discuss to what extent an analysis based on Feynman-Vernon can be extended to a system with a recovery map.

The general conditions for successful quantum error correction were formulated by Knill and Laflamme [40]. The starting point is a code space $\mathcal{C}$ of which the ground state of the Kitaev model, Sect. 8 in main text, is an example. The code space is a subset of a larger Hilbert space $\mathcal{H}$ called the coding space, and a super-operator $\Phi$ acts on density matrices on $\mathcal{H}$. Perfect quantum error correction in $\mathcal{C}$ under $\Phi$ is possible if there exist another superoperator $\mathcal{R}$, called a recovery operator, such that $\mathcal{R} \Phi$ acts as the identity on all pure states $|\Psi\rangle\langle\Psi|, \Psi \in \mathcal{C}$. The general form of $\mathcal{R}$ is that of a measurement of the component in $\mathcal{H}$ orthogonal to $\mathcal{C}$, followed by a unitary transformation. Alternatively, if $\Phi$ is represented in the Kraus form $\rho \rightarrow \sum_{k} A_{k} \rho A_{k}^{\dagger}$, the condition can be formulated as conditions on dynamical operators $A_{k}$ acting on $\mathcal{C}$ [40, Theorem III.2]. If some quantum dynamics $\Phi$ on some space $\mathcal{H}$ admits quantum error correction therefore reduces to the question if there exists a code space $\mathcal{C}$. In general this is not trivial to decide, see e.g. [45] for general rank-2 super-operators and [43] for general 2-qubit maps.

For error models often considered for the Kitaev code e.g. in [20] and [59] the above translates as follows. The coding space $\mathcal{H}$ is that of all the physical qubits, and $\Phi$ has a block structure where each block acts on the states of one physical qubit. Interaction with the environment has hence been assumed to lead to super-operator which is local in space (physical qubits) and also local in time (no memory), compare discussion in Sect. 4 in main text. By measuring stabilizers it is grosso modo possible to decide which unitary map was applied, and then correct for it by applying its inverse. Precision to this statement, consequences and concrete implementations have been discussed in great detail in the literature [20,25,38]. Within Feynman-Vernon theory the effects of random superposition of unitary transformations can be be described as follows: if the influence action from applying $\rho \rightarrow \rho^{\prime}=V_{a} \rho V_{a}^{\dagger}$ 
is $\Phi_{a}$, and if this transformation is applied with probability $p_{a}$, then the total influence action is $\frac{1}{i} \log \sum_{a} p_{a} e^{i \Phi_{a}}$ [24]. The total variational distance from (16) is then

$$
\mathrm{TVD} \approx \sum_{\mathbf{f}}\left|\sum_{a} p_{a}\left\langle\Phi_{a}\right\rangle_{i f}\right|
$$

If there are just a few unitary maps applied we are back to the same estimates as in Sect. 5, but if there are many and they contribute with random phases the resulting TVD could be smaller due to cancellations.

Making additional assumptions we can also discuss the recovery map in the Kitaev code with error correction in the Feynman-Vernon formalism. First, we assume that the stabilizers are measured very often but not absolutely continuously. This is in line with proposed hardware implementations based on a system clock [25], and implies that the degrees of the freedom of both the logical qubits and the stabilizers can change between measurements. Second, the record of all the measurements of all the stabilizers is assumed known. The corresponding chain of projection operators acting on the full density matrix of the logical qubits, the stabilizers and the environment is then a coarse-grained history in the sense of Gell-Mann and Hartle [26]. Third, these coarse-grained histories are assumed to fulfill the decoherence conditions of [26]. When (if) this is so we can consider the results of the measurements as known classical time-dependent parameters and write the Feynman-Vernon path integral for the logical qubits and the environment as in Sect. 8. The difference for the spin-boson terms would then be that while at most instance of time the appropriate form is (27) (when the measured values of the stabilizers do not change), but sometimes it is (30) (when they do).

\section{References}

1. Aaronson, S., Arkhipov, A.: The computational complexity of linear optics. Theory Comput. 9, 143-252 (2013). https://doi.org/10.4086/toc.2013.v009a004

2. Aaronson, S., Brod, D.J.: Bosonsampling with lost photons. Phys. Rev. A 93, 012,335 (2016). https:// doi.org/10.1103/PhysRevA.93.012335

3. Aharonov, D., Kitaev, A., Nisan, N.: Quantum circuits with mixed states. In: Proceedings of the Thirtieth Annual ACM Symposium on Theory of Computing, STOC '98, pp. 20-30. ACM, New York, NY, USA (1998). https://doi.org/10.1145/276698.276708

4. Alicki, R., Fannes, M., Horodecki, M.: A statistical mechanics view on kitaev's proposal for quantum memories. J. Phys. A Math. Theor. 40(24), 6451 (2007). http://stacks.iop.org/1751-8121/40/i=24/a=012

5. Alicki, R., Fannes, M., Horodecki, M.: On thermalization in kitaev's 2d model. J. Phys. A Math. Theor. 42(6), 065,303 (2009). http://stacks.iop.org/1751-8121/42/i=6/a=065303

6. Alicki, R., Horodecki, M., Horodecki, P., Horodecki, R.: Dynamical description of quantum computing: generic nonlocality of quantum noise. Phys. Rev. A 65, 062,101 (2002). https://doi.org/10.1103/ PhysRevA.65.062101

7. Atland, A., Simons, B.: Condensed Matter Field Theory. Cambridge University Press, Cambridge (2006)

8. Bapst, V., Foini, L., Krzakala, F., Semerjian, G., Zamponi, F.: The quantum adiabatic algorithm applied to random optimization problems: the quantum spin glass perspective. Phys. Rep. 523, 127-205 (2013)

9. Barenco, A., Bennett, C.H., Cleve, R., DiVincenzo, D.P., Margolus, N., Shor, P., Sleator, T., Smolin, J.A., Weinfurter, H.: Elementary gates for quantum computation. Phys. Rev. A 52, 3457-3467 (1995). https:// doi.org/10.1103/PhysRevA.52.3457

10. Bengtsson, I., Życzkowski, K.: Geometry of Quantum States. Cambridge University Press, Cambridge (2006)

11. Bombín, H.: Single-shot fault-tolerant quantum error correction. Phys. Rev. X 5, 031,043 (2015). https:// doi.org/10.1103/PhysRevX.5.031043

12. Bombín, H.: Resilience to time-correlated noise in quantum computation. Phys. Rev. X 6, 041,034 (2016). https://doi.org/10.1103/PhysRevX.6.041034 
13. Bombin, H., Chhajlany, R.W., Horodecki, M., Martin-Delgado, M.A.: Self-correcting quantum computers. New J. Phys. 15(5), 055,023 (2013). http://stacks.iop.org/1367-2630/15/i=5/a=055023

14. Bravyi, S., Hastings, M.B.: A short proof of stability of topological order under local perturbations. Commun. Math. Phys. 307(3), 609 (2011). https://doi.org/10.1007/s00220-011-1346-2

15. Bravyi, S., Terhal, B.: A no-go theorem for a two-dimensional self-correcting quantum memory based on stabilizer codes. New J. Phys. 11(4), 043,029 (2009). http://stacks.iop.org/1367-2630/11/i=4/a=043029

16. Breuer, H.P., Petruccione, F.: The Theory of Open Quantum Systems. Oxford University Press, Oxford (2002)

17. Caldeira, A., Leggett, A.: Path integral approach to quantum brownian motion. Physica A 121: 587-616 (1983). https://doi.org/10.1016/0378-4371(83)90013-4

18. Courtland, R.: Google plans to demonstrate the supremacy of quantum computing. IEEE Spectrum (2017)

19. Denchev, V.S., Boixo, S., Isakov, S.V., Ding, N., Babbush, R., Smelyanskiy, V., Martinis, J., Neven, H.: What is the computational value of finite-range tunneling? Phys. Rev. X 6, 031,015 (2016). https://doi. org/10.1103/PhysRevX.6.031015

20. Dennis, E., Kitaev, A., Landahl, A., Preskill, J.: Topological quantum memory. J. Math. Phys. 43(9), 4452-4505 (2002). https://doi.org/10.1063/1.1499754

21. Deutsch, D.: Quantum computational networks. Proc. R. Soc. Lond. A 425(1868), 73-90 (1989). https:// doi.org/10.1098/rspa.1989.0099

22. Devoret, M., Wallraf, A., Martinis, J.: Superconducting qubits: a short review. arXiv:cond-mat/0411174 (2004)

23. Feynman, R.P.: Simulating physics with computers. Int. J. Theor. Phys. 21(6/7), 467-488 (1982)

24. Feynman, R.P., Vernon, F.: The theory of a general quantum system interacting with a linear dissipative system. Ann. Phys. 24, 118-173 (1963)

25. Fowler, A.G., Mariantoni, M., Martinis, J.M., Cleland, A.N.: Surface codes: towards practical large-scale quantum computation. Phys. Rev. A 86, 032,324 (2012). https://doi.org/10.1103/PhysRevA.86.032324

26. Gell-Mann, M., Hartle, J.B.: Classical equations for quantum systems. Phys. Rev. D 47, 3345-3382 (1993). https://doi.org/10.1103/PhysRevD.47.3345

27. Georgescu, I.M., Ashhab, S., Nori, F.: Quantum simulation. Rev. Mod. Phys. 86, 153-185 (2014). https:// doi.org/10.1103/RevModPhys.86.153

28. Gershenfeld, N.A., Chuang, I.L.: Bulk spin-resonance quantum computation. Science 275(5298), 350356 (1997). https://doi.org/10.1126/science.275.5298.350

29. Ghirardi, G.C., Rimini, A., Weber, T.: Unified dynamics for microscopic and macroscopic systems. Phys. Rev. D 34, 470-491 (1986). https://doi.org/10.1103/PhysRevD.34.470

30. Grifoni, M., Hänggi, P.: Driven quantum tunneling. Phys. Rep. 304(5), 229-354 (1998). https://doi.org/ 10.1016/S0370-1573(98)00022-2

31. Haroche, S., Raimond, J.M.: Quantum computing: dream or nightmare? Phys. Today 49, 51-52 (1996)

32. Harrow, A.W., Montanaro, A.: Quantum computational supremacy. Nature 549, 203 (2017). https://doi. org/10.1038/nature23458

33. Kalai, G.: Quantum computers: noise propagation and adversarial noise models. arXiv:0904.3265 (2009)

34. Kalai, G.: The quantum computer puzzle. Not. AMS 63(5), 508-516 (2016)

35. Kalai, G.: The quantum computer puzzle (expanded version). arXiv:1605.00992 (2016)

36. Kalai, G., Kindler, G.: Gaussian noise sensitivity and bosonsampling. arXiv:1409.3093 (2014)

37. Kelly, J., Barends, R., Fowler, A.G., Megrant, A., Jeffrey, E., White, T.C., Sank, D., Mutus, J.Y., Campbell, B., Chen, Y., Chen, Z., Chiaro, B., Dunsworth, A., Hoi, I.C., O’Malley, P.J.J., Neill, C., Quintana, C., Roushan, P., Vainsencher, A., Wenner, J., Cleland, A.N., Martinis, J.M.: State preservation by repetitive error detection in a superconducting quantum circuit. Nature 519, 66-69 (2015). https://doi.org/10.1038/ nature 14270

38. Kitaev, A.Y.: Quantum computations: algorithms and error correction. Russ. Math. Surv. 52(6), 1191 (1997). http://stacks.iop.org/0036-0279/52/i=6/a=R02

39. Klauder, J.R.: Path integrals and stationary-phase approximations. Phys. Rev. D 19, 2349-2356 (1979). https://doi.org/10.1103/PhysRevD.19.2349

40. Knill, E., Laflamme, R.: Theory of quantum error-correcting codes. Phys. Rev. A 55, 900-911 (1997). https://doi.org/10.1103/PhysRevA.55.900

41. Ladd, T.D., Jelezko, F., Laflamme, R., Nakamura, Y., Monroe, C., OBrien, J.L.: Quantum computers. Nature 464, 45-53 (2010). https://doi.org/10.1038/nature08812

42. Leggett, A.J., Chakravarty, S., Dorsey, A.T., Fisher, M.P.A., Garg, A., Zwerger, W.: Dynamics of the dissipative two-state system. Rev. Mod. Phys. 59, 1-85 (1987). https://doi.org/10.1103/RevModPhys.59. 1

43. Lipka-Bartosik, P., Życzkowski, K.: Nuclear numerical range and quantum error correction codes for nonunitary noise models. Quantum Inf. Process. 16: 9 (2017). https://doi.org/10.1007/s11128-016-1484-8 
44. Lund, A.P., Bremner, M.J., Ralph, T.C.: Quantum sampling problems, bosonsampling and quantum supremacy. npj Quantum Inf. 3, 15 (2017). https://doi.org/10.1038/s41534-017-0018-2

45. Majgier, K., Maassen, H., Życzkowski, K.: Protected subspaces in quantum information. Quantum Inf. Process. 9: 343-367 (2010). https://doi.org/10.1007/s11128-009-0131-z

46. Mermin, N.D.: Quantum Computer Science: An Introduction. Cambridge University Press, Cambridge (2007)

47. Michał, Brod, D.: Classical simulation of photonic linear optics with lost particles. arXiv:1801.06166 (2018)

48. Mirrahimi, M., Leghtas, Z., Albert, V.V., Touzard, S., Schoelkopf, R.J., Jiang, L., Devoret, M.H.: Dynamically protected cat-qubits: a new paradigm for universal quantum computation. New J. Phys. 16(4), 045,014 (2014). http://stacks.iop.org/1367-2630/16/i=4/a=045014

49. Nielsen, M.A., Chuang, I.L.: Quantum Computation and Quantum Information. Cambridge University Press, Cambridge (2000)

50. Nikolopoulos, G.M., Brougham, T.: Decision and function problems based on boson sampling. Phys. Rev. A 94, 012315 (2016). https://doi.org/10.1103/PhysRevA.94.012315

51. Paik, H., Schuster, D.I., Bishop, L.S., Kirchmair, G., Catelani, G., Sears, A.P., Johnson, B.R., Reagor, M.J., Frunzio, L., Glazman, L.I., Girvin, S.M., Devoret, M.H., Schoelkopf, R.J.: Observation of high coherence in josephson junction qubits measured in a three-dimensional circuit qed architecture. Phys. Rev. Lett. 107, 240501 (2011). https://doi.org/10.1103/PhysRevLett.107.240501

52. Paladino, E., Galperin, Y.M., Falci, G., Altshuler, B.L.: $1 / f$-noise: implications for solid-state quantum information. Rev. Mod. Phys. 86, 361-418 (2014). https://doi.org/10.1103/RevModPhys.86.361

53. Preskill, J.: Quantum computing and the entanglement frontier. arXiv:1203.5813 (2012)

54. Preskill, J.: Sufficient condition on noise correlations for scalable quantum computing. Quant. Inf. Comput. 13, 181-194 (2013)

55. Prokof'ev, N.V., Stamp, P.C.E.: Theory of the spin bath. Rep. Progr. Phys. 63(4), 669 (2000). http://stacks. iop.org/0034-4885/63/i=4/a=204

56. Savage, N.: Quantum Computers Compete for "Supremacy". Scientific American, New York (2017)

57. Schulman, L.S.: Techniques and Applications of Path Integration. Wiley, New York (1981)

58. Stone, M.: Supersymmetry and the quantum mechanics of spin. Nucl. Phys. B 314, 577-586 (1989)

59. Terhal, B.M.: Quantum error correction for quantum memories. Rev. Mod. Phys. 87, 307-346 (2015). https://doi.org/10.1103/RevModPhys.87.307

60. Terhal, B.M., Burkard, G.: Fault-tolerant quantum computation for local non-markovian noise. Phys. Rev. A 71, 012,336 (2005). https://doi.org/10.1103/PhysRevA.71.012336

61. Troyansky, L., Tishby, N.: Permanent uncertainty: On the quantum evaluation of the determinant and the permanent of a matrix. In: Proceedings of the 4th Workshop on Physics and Computation. New England Complex Systems Institute (1996). https://www.cs.huji.ac.il/labs/learning/Papers/perm.pdf

62. Weinberg, S.: Collapse of the state vector. Phys. Rev. A 85, 062,116 (2012). https://doi.org/10.1103/ PhysRevA.85.062116

63. Weinberg, S.: Lindblad decoherence in atomic clocks. Phys. Rev. A 94, 042,117 (2016). https://doi.org/ 10.1103/PhysRevA.94.042117

64. Weinberg, S.: The Trouble with Quantum Mechanics. New York Review of Books, New York (2017)

65. Wendin, G., Shumeiko, V.: Superconducting Quantum Circuits, Qubits and Computing, vol. 3 (2004)

66. Wheeler, J.A., Zurek, W.H. (eds.): Quantum Theory and Measurement. Princeton University Press, Princeton (2014)

67. Zurek, W.H.: Decoherence, einselection, and the quantum origins of the classical. Rev. Mod. Phys. 75, 715-775 (2003). https://doi.org/10.1103/RevModPhys.75.715 